\section{Transcriptome Analysis, Marker Discovery and Pigment Biosynthesis of Red-leaf Juglans regia}

\author{
Xin Chen \\ Shandong Institute of Pomology, Shandong Provincial Key Laboratory of Fruit Tree \\ Biotechnology Breeding, Tai'an, Shandong, China
}

$\mathrm{Li} \mathrm{Xu}$

Shandong Institute of Pomology, Shandong Provincial Key Laboratory of Fruit Tree Biotechnology Breeding, Tai'an, Shandong, China

\section{Xiao-juan Zong}

Shandong Institute of Pomology, Shandong Provincial Key Laboratory of Fruit Tree Biotechnology Breeding, Tai'an, Shandong, China

\section{Hai-rong Wei}

Shandong Institute of Pomology, Shandong Provincial Key Laboratory of Fruit Tree Biotechnology Breeding, Tai'an, Shandong, China

Jia-wei Wang

Shandong Institute of Pomology, Shandong Provincial Key Laboratory of Fruit Tree Biotechnology Breeding, Tai'an, Shandong, China

\section{Dong-zi Zhu}

Shandong Institute of Pomology, Shandong Provincial Key Laboratory of Fruit Tree Biotechnology Breeding, Tai'an, Shandong, China 
Yue Tan

Shandong Institute of Pomology, Shandong Provincial Key Laboratory of Fruit Tree

Biotechnology Breeding, Tai'an, Shandong, China

\title{
Mao-run Fu
}

College of Food Science and Engineering, Qilu University of Technology, Jinan, China

\author{
Qing-zhong Liu (Corresponding author)
}

Shandong Institute of Pomology, No. 66 Longtan Road, Taian, China

Received: March 1, 2017 Accepted: May 28, 2017 Published: June 4, 2017

Doi: 10.5296/jab.v5i2.11345 URL: http://doi.org/10.5296/jab.v5i2.11345

\begin{abstract}
Red-leaf trait rarely occurs in Juglans regia, and the genetic mechanism underlying this phenomenon is still unknown. In this study, we attempted to provide insight into the comprehensive transcriptome of red-leaf $J$. regia by RNA-Seq using Illumina SeqTM2000 platform. A total of 33,488,602 high-quality reads (3.35G cleans bases) were obtained and assembled into 53,782 unigenes. A total of 3,683 unigenes were annotated by using basic local alignment search tool to search against protein databases, All the matched unigenes were categorized by gene ontology analysis, and 3,466 were assigned to metabolism, among which 74 were mapped to anthocyanin, carotenoid, and betalain biosynthetic pathways by Kyoto Encyclopedia of Genes and Genomes analysis. Approximately 656 transcription factors were isolated including MYB, NAC, and bHLH. Additionally, a total of 13,981 simple sequence repeats, 41,088 single nucleotide polymorphisms, and 5,860 insertions and deletions were determined from $J$. regia transcriptome. Therefore, the current $J$. regia transcriptome provides deep insight into the molecular basis of red-leaf breeding of J. regia.
\end{abstract}

Keywords: Juglans regia, Red-leaf, Marker discovery, Pigment biosynthesis

\section{Introduction}

Walnut (Juglans regia) belongs to the Juglans genus of Juglandaceae family and is a native to the region from Balkans eastward to the Himalayas and southwest China; this plant is now widely spread in Asia, America, and southern and eastern Europe (Nael Abu Taha, 2011). J. regia is a deciduous tree; its fruits are nutritional nuts rich in unsaturated fatty acids, tocopherols, and phytosterols (Amaral et al., 2008), and its leaves have been used as traditional medicine for the treatment of diabetes mellitus, skin inflammations, toothache, 
venous insufficiency, and ulcers (Hosseini et al., 2014b; Paudel et al., 2013). J. regia leaves reportedly have antioxidative, antimicrobial, antihypertensive, antihelmintic, and hypoglycemic effects (Hosseini et al., 2014a; Nael Abu Taha, 2011; Pereira et al., 2007; Qureshi et al., 2014; Zhao et al., 2014).

Different colors are determined by different kinds of plant pigments. Generally, green plants contain abundant chlorophyll, as well as carotenoids and anthocyanin. Among these components, chlorophyll is predominant and is responsible for the green color of plant leaves. However, red-colored leaves naturally exist in some plants, such as Gossypium hirsutum, Cotinus coggygria, Brassica oleracea, and Prunus cerasifera (Cai et al., 2014; Wang et al., 2013). The content of each compound determines the different colors of plant leaves. A high amount of chlorophyll makes leaves green, and a low amount makes them red. The red pigmentation of G. hirsutum L. leaf, crabapple leaf, and Jatropha curcas (L.) new leaf were reportedly induced by anthocyanin accumulation (Ranjan et al., 2014; Tian et al., 2015). In the red leaves of Sorghum bicolor, carotenoids are the predominant compounds, followed by flavonoids and phenolic acids; a small amount of chlorophyll (a and b) was also present in $S$. bicolor red leaves (Abugri et al., 2013). The ever-red leaf trait rarely occurs in the Juglans genus, thereby providing us a novel material for leaf color study. The red coloration of leaf is an attractive feature for ornamental value, and the compounds contributing to red coloration in leaves have biological and ecological importance. For example, anthocyanin can protect plants against pathogens and insects and can attract insect pollinators (Mouradov and Spangenberg, 2014); carotenoids participate in photosynthesis, photomorphogenesis, and photoprotection. However, information on the molecular mechanism underlying pigment biosynthesis in red leaves of $J$. regia is lacking. In 2012, Wu et al., performed the genome sequencing of $J$. regia by bacterial artificial chromosome (BAC) technology, yielding 31.2 $\mathrm{Mb}$ bases which accounted for only $5.1 \%$ of the whole genome( $\mathrm{Wu}$ et al., 2012). And the whole-genome of $J$. regia was sequenced by the end of 2014, however the data was still not available for public. More recently, next-generation sequencing (NGS) is a cost-efficient way to apply in gene discovery and analysis of gene expression for non-model species without reference genome.

Generally, pigment biosynthesis in plants is controlled by structural genes in pigment biosynthetic pathways and regulatory genes, e.g., transcription factors (TFs). The aim of this study was to explore molecular markers and pigment biosynthesis of the red-leaf trait by NGS method.

\section{Methods}

\subsection{Sample Collection and Preparation}

The red leaves of $J$. regia were collected from National Clonal Plant Germplasm Repository, Taian, Shandong Province, China. Total RNA was isolated from J. regia leaves using Trizol (Invitrogen) and was treated with DNase I to remove the genomic DNA. RNA degradation and contamination were checked on $1 \%$ agarose gels. RNA purity was assessed using the NanoPhotometer ${ }^{\circledR}$ spectrophotometer (IMPLEN, CA, USA). RNA concentration was measured using Qubit ${ }^{\circledR}$ RNA Assay Kit in Qubit ${ }^{\circledR}$ 2.0 Flurometer (Life Technologies, CA, 
USA), and RNA integrity (RIN) was quantified using the RNA Nano 6000 Assay Kit of the Bioanalyzer 2100 system (Agilent Technologies, CA, USA).

\subsection{Transcriptome Sample Preparation for Sequencing}

A total amount of $3 \mu \mathrm{g}$ RNA per sample was used for sample preparation. All prepared RNA samples had RIN values above 8. Sequencing libraries were generated using Illumina TruSeq $^{\text {TM }}$ RNA Sample Preparation Kit (Illumina, San Diego, USA) following manufacturer's recommendations, and index codes were added to attribute sequences to each sample. The mRNA was purified from total RNA using poly-T oligo-attached magnetic beads. Fragmentation was performed using divalent cations under elevated temperature in Illumina proprietary fragmentation buffer. First strand cDNA was synthesized using random oligonucleotides and SuperScript II. Second strand cDNA synthesis was subsequently performed using DNA polymerase I and RNase H. Remaining overhangs were converted into blunt ends via exonuclease/polymerase activities, and the enzymes were removed. After adenylation of the 3' ends of DNA fragments, Illumina PE adapter oligonucleotides were ligated to prepare for hybridization. To select cDNA fragments that are preferentially 200 base pair (bp) in length, the library fragments were purified with AMPure XP system (Beckman Coulter, Beverly, USA). DNA fragments with ligated adapter molecules on both ends were selectively enriched using Illumina PCR Primer Cocktail in a 10-cycle PCR reaction. Products were purified (AMPure XP system) and quantified using the Agilent high sensitivity DNA assay on the Agilent Bioanalyzer 2100 system.

\subsection{Clustering and Sequencing}

The clustering of the index-coded samples was performed on a cBot Cluster Generation System using TruSeq PE Cluster Kit v3-cBot-HS (Illumina) according to the manufacturer's instructions. After cluster generation, the library preparations were sequenced on an Illumina Hiseq 2000 platform, and $90 \mathrm{bp}$ paired-end reads were generated.

\subsection{Analysis of Illumina Sequencing Data}

Raw reads were initially processed through in-house perl scripts, and the clean reads were obtained by removing reads containing adapter and poly- $\mathrm{N}$ and low quality reads. All downstream analyses were based on high-quality clean reads. The transcriptome assembly was accomplished using Trinity software (Grabherr et al., 2011). Gene function was annotated after sequences were searched against the following databases: NCBI non-redundant protein sequences $(\mathrm{Nr})$, NCBI non-redundant nucleotide sequences $(\mathrm{Nt})$, Protein family (Pfam), Clusters of Orthologous Groups of proteins (KOG), Swiss-Prot (A manually annotated and reviewed protein sequence database), Kyoto Encyclopedia of Genes and Genomes (KEGG) Ortholog database (KO), and Gene Ontology (GO). To discover single nucleotide polymorphisms (SNPs), Picard-tools v1.41 (http://picard.sourceforge.net) and Samtools v0.1.18 (http://sourceforge.net/projects/samtools/files/samtools/) were used to sort and remove duplicated reads and merge the bam alignment results of each sample. GATK2 software was used to perform SNP calling, and only SNPs with distances higher than 5 were retained with the GATK standard filter method (McKenna et al., 2010). Simple sequence 


\section{MInstitute Macrothink $_{\text {Int }}$}

repeats (SSRs) of the transcriptome were identified using microsatellite identification tool. Gene expression patterns were estimated by RNA-Seq by Expectation-Maximization (RSEM). First, clean data were mapped back to the assembled transcriptome, and the read counts for each gene were achieved from the mapping results. Finally, the read counts were normalized into Reads Per Kilo bases per Million mapped Reads (RPKM).

\section{Results and Discussions}

\subsection{Assembled transcriptome}

To obtain an overview of comprehensive transcripts of $J$. regia red leaves, a cDNA library of J. regia red leaves was generated and pair end sequenced using Illumina HisSeqTM2000 platform, thereby generating 34,634,887 raw reads after base calling. Generally, the error rate of each sequenced base should be below $1 \%$, and error rate would increase with increasing sequence read length. The error rate distribution is shown in Figure 1. After removing adapter-related sequences, low quality reads and reads containing N, 33,488,602 clean reads were obtained with $3.35 \mathrm{G}$ clean bases in total, and the clean reads were used for bioinformatics analysis. All clean reads were assembled into 100,605 transcripts using Trinity software, with mean length of 1,200 bp. The longest transcript of each gene was defined as a unigene, and 53,782 unigenes (including 42,966,265 nucleotides) were obtained with a mean length of 799 bp (Table 1). The entire transcriptome (SRA accession Number SRP054989) assembled was used as reference sequences for further analysis. The length distributions of all transcripts and unigenes are shown in Additional File 1.

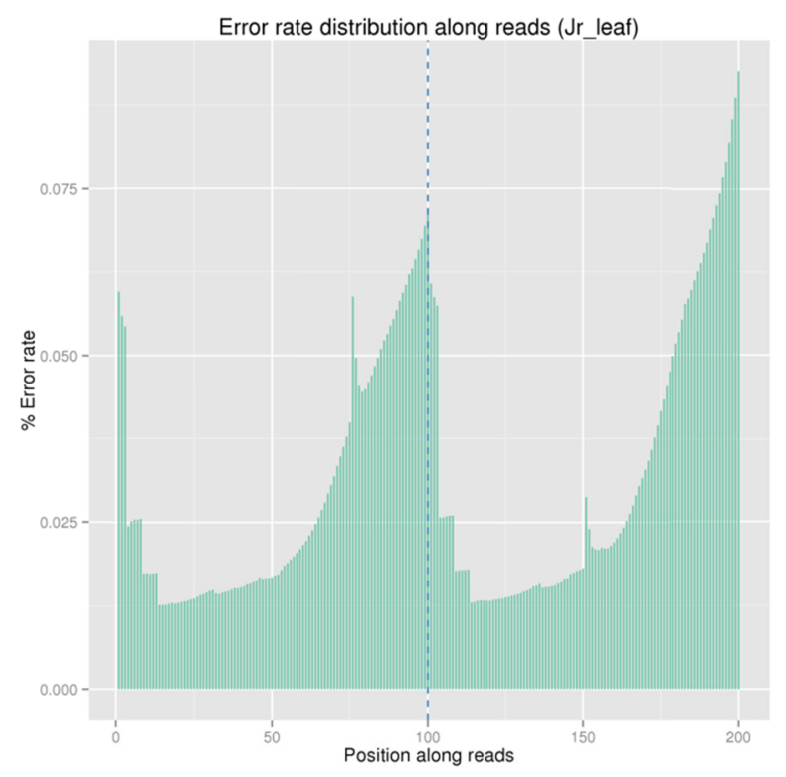

Figure 1. Error rate distribution along reads of J. regia transcriptome 
Table 1 . Summary of $J$. regia Transcriptome and de novo assembly

$\begin{array}{llll}\text { Raw } & \text { Clean } & \text { Clean } & \text { Error } \\ \text { Reads } & \text { Reads } & \text { Bases } & (\%)\end{array}$

\begin{tabular}{llllllllll}
\hline Numbers/Rate & 34634887 & 33488602 & $3.35 \mathrm{G}$ & 0.03 & & & \\
& $\begin{array}{l}\text { Min } \\
\text { length }\end{array}$ & $\begin{array}{l}\text { Mean } \\
\text { length }\end{array}$ & $\begin{array}{l}\text { Median } \\
\text { length }\end{array}$ & $\begin{array}{l}\text { Max } \\
\text { length }\end{array}$ & N50 & N90 & $\begin{array}{l}\text { Total } \\
\text { nucleotides }\end{array}$ \\
Transcripts & 201 & 1200 & 813 & 14850 & 1984 & 516 & 120691294 \\
Unigenes & 201 & 799 & 398 & 14850 & 1532 & 294 & 42966265 \\
\hline
\end{tabular}

\subsection{Gene Function Annotation}

For functional annotation of $J$. regia transcriptome, all unigenes were searched against seven public databases. Using this approach, 28,351 unigenes $(52.71 \%$ of all unigenes) returned a significant basic local alignment search tool result. Among these unigenes, 3,683 (6.84\%) were annotated in all databases (Table 2). Approximately $47.29 \%$ of the total unigenes had no homology in these databases, thereby suggesting that these transcripts may be unique to $J$. regia.

Table 2. Statistics of annotation percentages of J. regia unigenes in public databases

\begin{tabular}{lll}
\hline & Number of Unigenes & Percentage (\%) \\
\hline Annotated in NR & 26315 & 48.92 \\
\hline Annotated in NT & 14573 & 27.09 \\
\hline Annotated in KO & 7505 & 13.95 \\
\hline Annotated in SwissProt & 18289 & 34 \\
\hline Annotated in PFAM & 17257 & 32.08 \\
\hline Annotated in GO & 19934 & 37.06 \\
\hline Annotated in KOG & 8969 & 16.67 \\
\hline Annotated in all Databases & 3683 & 6.84 \\
\hline
\end{tabular}


Annotated in at least one 28351

52.71

Database

Total Unigenes

53782

100

GO is a classification system that can describe the biological process (BP), cellular component (CC), and molecular function (MF) of genes. For classification of gene functions, GO assignment was performed. A total of 19,934 unigenes were assigned to one or more GO terms, and all these 19,934 unigenes were categorized into 65 functional groups, which are distributed to three main categories, as follows: BP $(53,093)$, MF $(25,579)$, and CC $(37,311)$ (Figure 2). However, the categories with no more than 20 unigenes are not shown in Figure 1. Within the BP category, cellular (12290 unigenes, 23.15\%), metabolic (11688 unigenes, $22.01 \%$ ), and single-organism (5790 unigenes, 10.90\%) processes were the most enriched. Under the CC category, cell (7693 unigenes, 20.62\%), cell part (7679, 20.58\%), and organelle (5279 unigenes, $14.15 \%$ ) were the most highly represented GO terms. In the MF category, the majority of unigenes were involved in binding (11,647 unigenes, 45.53\%), catalytic activity $(10,107$ unigenes, $39.51 \%)$, and transporter activity (1,438 unigenes, $5.62 \%)$.

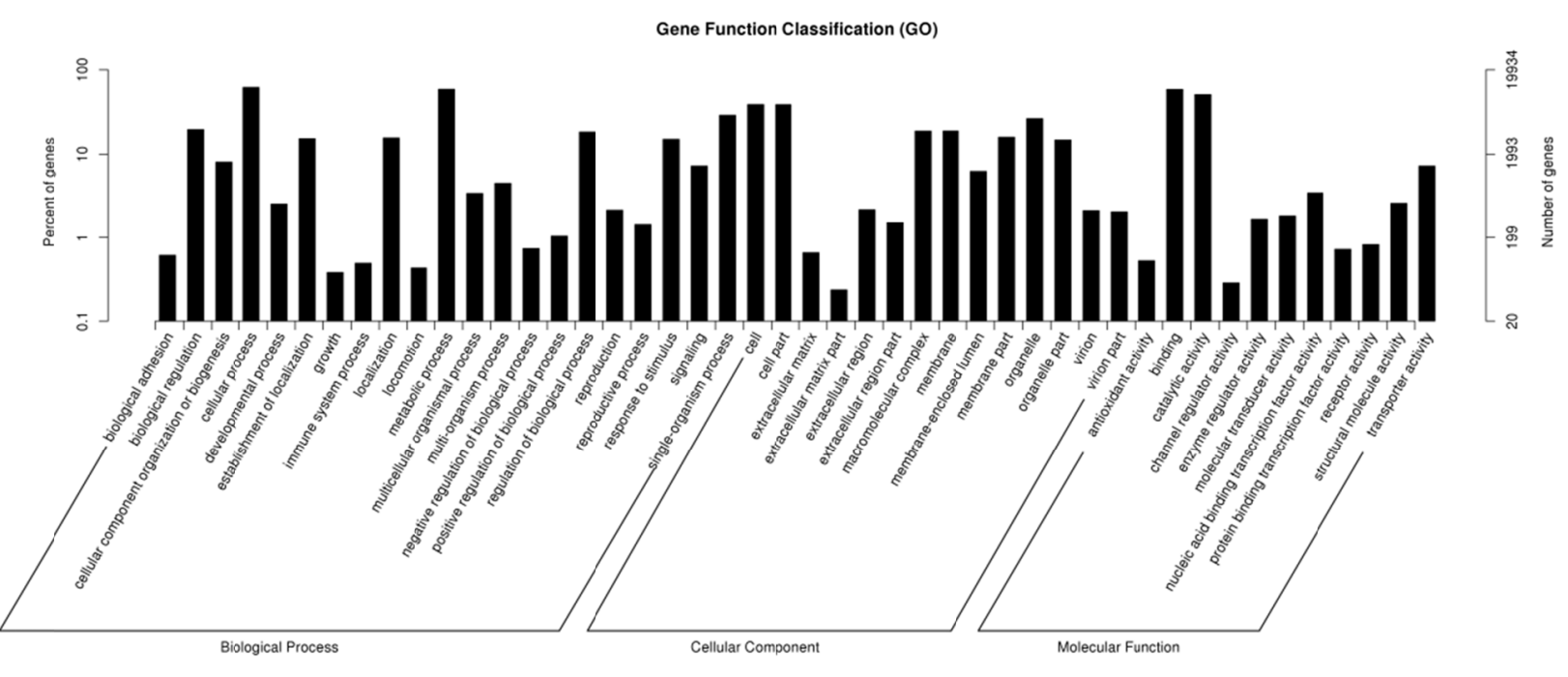

Figure 2. GO Classification of $J$. regia unigenes

The three main categories of GO terms were BP, CC, and MF. The horizontal axis was a GO term, and the vertical axis represented the number and percentage of annotated unigenes. The categories with numbers below 20 are not shown in this Figure.

EuKaryotic Ortholog Groups (KOG) analysis was used to further evaluate the functions of annotated unigenes specific to eukaryons. A total of 8,969 unigenes were annotated into 26 


\section{Macrothink}

KOG groups, the cluster of general function prediction only (1,610 unigenes, 17.95\%) was the largest group, followed by post-translational modification, protein turnover, chaperon (1,207 unigenes, 12.90\%), and signal transduction (822 unigenes, 9.16\%). The unnamed protein ( 1 unigenes, $0.00 \%$ ), cell motility ( 3 unigenes, $0.00 \%$ ), and extracellular structures (30 unigenes, $0.33 \%$ ) represented the smallest groups (Figure 3 ).

KOG Classification

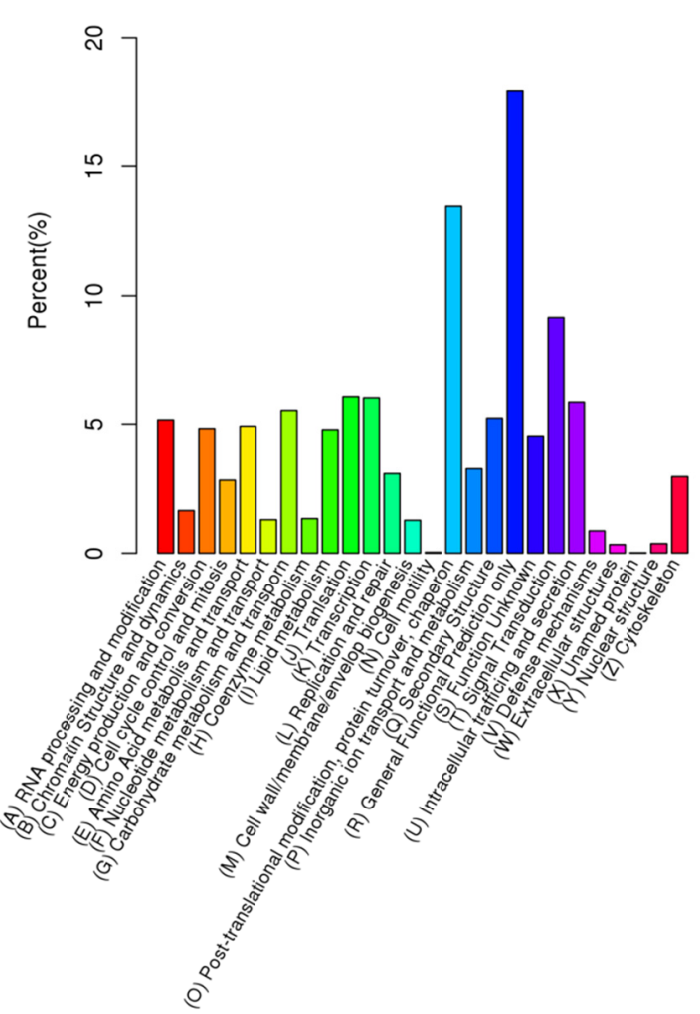

Figure 3. KOG Classification of J. regia unigenes

These 8,969 unigenes of $J$. regia were assigned into $26 \mathrm{KOG}$ categories. The $\mathrm{x}$-axis comprised KOG categories, whereas the $\mathrm{y}$-axis comprised percentage of assigned unigenes.

After $\mathrm{KO}$ annotation analysis, we further classified the unigenes based on the KEGG metabolic pathways. KEGG pathways cover the six main groups, such as metabolism, genetic information processing, environmental information processing, cellular processes, organismal systems, and human diseases. In total, 7,505 unigenes were mapped to 241 KEGG pathways. In addition, we created a statistical data of unigenes involved in the second hierarchy pathways without human diseases category, and the categories showing the greatest representation by unigenes were metabolism (3,466 unigenes) and organismal systems $(1,235$ unigenes) (Figure 4). Among all the related secondary metabolism pathways, biosyntheses of anthocyanin (2 unigenes), flavone and flavonol (10 unigenes), flavonoid (32 unigenes), and 


\section{Macrothink}

carotenoid (28 unigenes) were associated with color formation and variation.

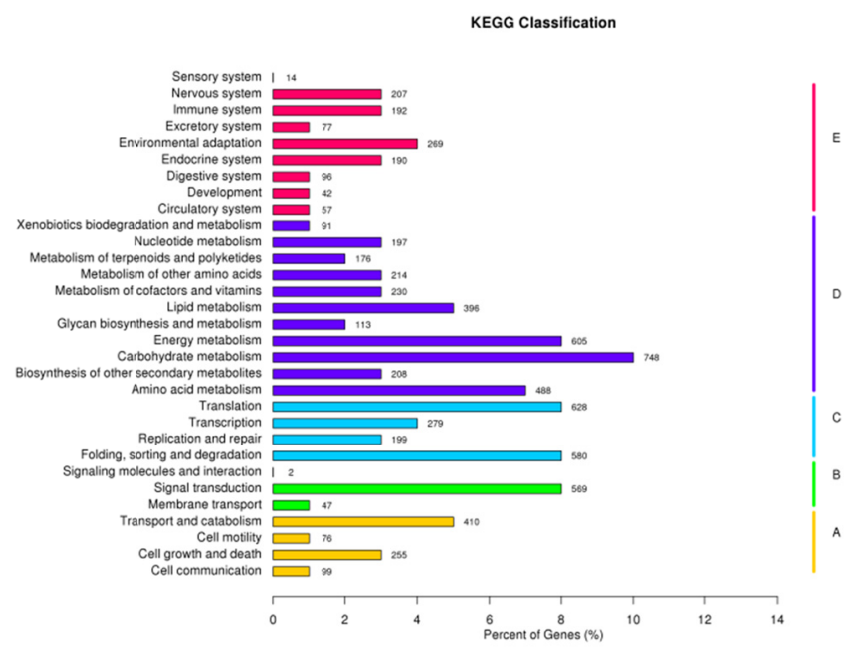

Figure 4. KEGG Classifications of $J$. regia unigenes

A: Cellular Processes; B: Environmental Information Processing; C: Genetic Information Processing; D: Metabolism; E: Organismal Systems.

\subsection{SSR Discovery in The J. regia Transcriptome Assembly}

SSRs are short tandem repeat sequences (1-6 bp) and are distributed throughout the genome. For the past 20 years, SSRs have been widely applied for plant genetic analysis because of their highly polymorphic characterization. From the 53,782 unigenes that were examined in $J$. regia transcriptome assembly, 11,315 unigenes containing putative SSRs were identified with the occurrence frequency of $21.04 \%$, among which 2,148 unigenes had more than one SSR marker signature. A total of 13,981 SSRs were discovered, of which 815 SSRs were of compound formation (Table 3). Repeat motifs among these SSRs consisted of 69 types, and the number of repeat unit size varied from 5 to 24 . Table 4 shows the density of different types of SSRs. The single base repeats were the most abundant motifs (49.29\%), followed by dibase type SSRs (37.42\%). In recent years, several SSR markers have been developed by screening expressed sequencing tags (ESTs). Thirty-five and 30 highly transferable and polymorphic expressed sequencing tags-simple sequence repeats (EST-SSRs) and 706 EST-SSRs were identified in J. regia by Qi et al. (2010), Zhang et al. (2010), and Zhang et al. (2013), respectively (Qi JX, 2011; Zhang R, 2010; Zhang et al., 2013). Therefore, compared with the previous results, our study obtained more SSRs. Moreover, the new SSRs would be further identified by primer designing and PCR reactions. 
Table 3. Summary of SSR Analysis in the J. regia Transcriptome

Total number of sequences examined 53782

Total size of examined sequences (bp) 42966265

Total number of identified SSRs

13981

Number of SSR containing sequences 11315

Number of sequences containing more 2148 than 1 SSR

Number of SSRs present in compound 815 formation

Table 4. Frequency of different SSR types in the J. regia transcriptome

\begin{tabular}{|c|c|c|c|c|c|c|c|c|c|c|}
\hline \multirow{2}{*}{$\begin{array}{l}\text { SSRs } \\
\text { type }\end{array}$} & \multicolumn{9}{|c|}{ Repeat Number } & \multirow[t]{2}{*}{ Total } \\
\hline & 5 & 6 & 7 & 8 & 9 & 10 & 11 & $\begin{array}{l}>11 \\
\leq 20\end{array}$ & $>20$ & \\
\hline P1 & - & - & - & - & - & 2037 & 1241 & 3419 & 194 & 6891 \\
\hline P2 & - & 1254 & 924 & 950 & 1150 & 792 & 154 & 7 & - & 5231 \\
\hline P3 & 881 & 457 & 314 & 20 & - & - & - & 3 & 1 & 1676 \\
\hline P4 & 118 & 29 & 2 & 1 & - & - & - & 1 & - & 151 \\
\hline P5 & 20 & - & - & - & - & - & - & 1 & - & 21 \\
\hline P6 & 5 & 4 & - & - & 1 & - & - & 1 & - & 11 \\
\hline Total & 1024 & 1744 & 1240 & 971 & 1151 & 2829 & 1395 & 3432 & 195 & 13981 \\
\hline
\end{tabular}




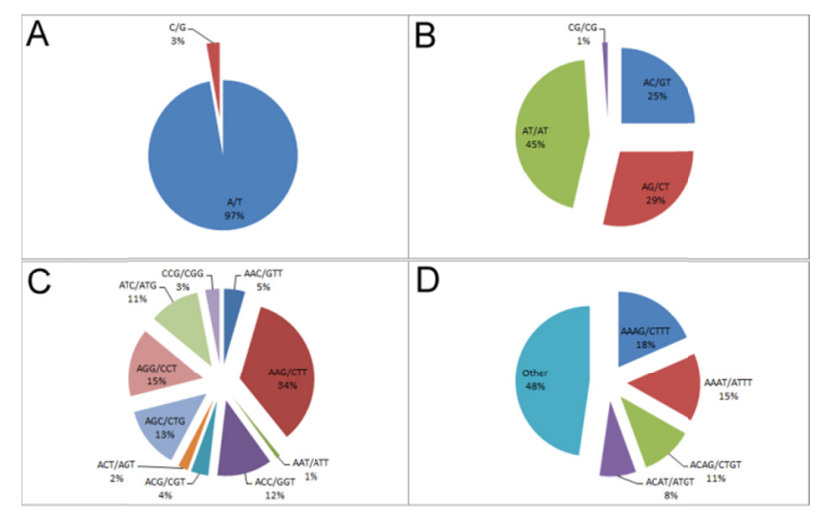

Figure 5. Percentages of Different Motifs among Single-nucleotide (A), Dinucleotide (B), Tribucleotide (C), and Tetranucleotide (D) in the J. regia Transcriptome

\subsection{Identification of SNP and InDel Polymorphisms}

Although SSR exploration has been conducted in J. regia, SSR frequency was lower than SNP (Kaya et al., 2013). A total of 41,088 SNPs and 5,860 insertion/deletion polymorphisms (InDels) were detected. Of the 5,860 InDels, 4616 were insertions and 1,244 were deletions. Of the 41,088 SNPs, 8,424 were transitions and 32,652 were transversions (with a ratio of approximately $1: 4)$.

\subsection{Mining of Genes are Putatively Related to Leaf Red-pigment Trait}

Generally, chlorophyll makes leaf green, and flavonoids, flavone and flavonol, anthocyanin, betalain, and carotenoid biosynthesis are related to red color pigment (Tanaka et al., 2008). In $J$. regia transcriptome, nearly all genes involved in chlorophyll biosynthesis were found. The flavonoid compounds were the major pigments responsible for colors of flower and non-green leaves, and the ratio of different anthocyanidins resulted in different colors. To date, we are not sure which pigments were involved in red mutation because of the lack of pigment content determination in $J$. regia leaves, which may be the focus of further study. Therefore, we analyzed related genes in the biosynthesis of the pigments mentioned above. From $J$. regia transcriptome, genes involved in these pathways were analyzed and searched by standard gene names and synonyms (Additional File 2).

A total of 74 unigenes (including 50 genes) were assigned to the biosynthetic pathways above by mapping to KEGG pathways. Anthocyanins, a class of flavonoids, are synthesized in the cytosol and stored in the vacuole. The colors of anthocyanins ranged from red to blue depending on $\mathrm{pH}$, metal ions, and co-pigments (typically flavones and flavonols). In a previous study by Zhao et al., 17 compounds isolated and identified from the leaves of $J$. regia were collected from Tianjin, China (Zhao et al., 2014). Among these unigenes, all genes except for four annotated in flavonoid biosynthetic pathway were found (Additional file 3A). Additionally, in the anthocyanin biosynthesis of the downstream, all genes were identified 
except for the last gene encoding UDP glucose: flavonoid 3-Oglucosyltransferase (3B). Ten genes were in the flavone and flavonol pathways (3C). Carotenoids are terpenoid compounds with colors ranging from yellow to red and are synthesized in chloroplasts. All genes involved in carotene biosynthesis and most genes involved in lutein and astaxanthin (carotenoid) biosynthesis were identified (Additional File 3D). Betalains are compounds responsible for violet and yellow color in plants, but the biosynthetic pathway has not been elucidated well. From the $J$. regia transcriptome, we found the key enzyme annotated as '4,5-DOPA extradiol dioxygenase' (Additional File 2E). Unlike anthocy/anin, betalains are more stable, and the color does not depend on the $\mathrm{pH}$ (Tanaka et al., 2008). We speculate that flavonoids and carotenoid may be responsible for the red color of J. regia leaves.

\subsection{Identification of TFS}

In previous studies, a set of TFs, including R2R3-MYB, basic helix-loop-helix (bHLH), and WD40-repeat protein, were identified as key regulators for transcription expression in pigment biosynthetic pathways (Grotewold, 2006; Jung et al., 2009; Yang et al., 2015; Zhu et al., 2014). A total of 656 unigenes annotated as TFs were found (Additional File 3), and the TF families with the largest number of unigenes were WRKY (64 unigenes), NAC (50 unigenes), bHLH (50 unigenes), MYB (49 unigenes), and heat shock factors (45 unigenes) (Table 5). TFs from MYB family were the most widespread regulators of anthocyanin biosynthesis. Tian et al. demonstrated that McMYB10 can positively regulate anthocyanin biosynthesis via flavonoid 3'-hydroxylase in ever-red leaf crabapple (Tian et al., 2015). In 2015, Albert et al. isolated two R2R3-MYB genes, RED LEAF and RED $V$, which were confirmed to be anthocyanin regulators (Albert et al., 2015). Zhou et al. found that overexpression of PpMYB10.4 induces anthocyanin accumulation in tobacco and peach leaves (Zhou et al., 2014). Other evidences support that carotenoids biosynthesis were mainly regulated at the transcriptional level (Sandmann et al., 2006). In tomato, SINAC4 functioned as a positive regulator of carotenoid accumulation (Zhu et al., 2014). Moreover, several unigenes encoding phytochrome-interacting factors, a class of bHLH TFs that can increase carotenoids accumulation (Rodriguez-Villalon et al., 2009), were also identified in our $J$. regia transcriptome. The identified TFs potentially involved in pigment biosynthesis regulation can be explored deeply in the future.

Table 5. Statistics of transcription factors in red-leaf $J$. regia

Transcription factors Number

WRKY

bHLH

50 


\section{MYB}

Heat shock

GATA

MADS-box

AP2/ERF

WD-repeat

GRAS

AP2 domain containing

R2R3-MYB

HD-domain
49

45

10

\section{1}

15

15

13

\subsection{Analysis of Gene Expression Level}

The $J$. regia transcriptome assembled by Trinity was used for analyzing reference sequences, and of all 33,488,602 clean reads, 28,493,056 (85.08\%) were mapped to the reference transcriptome. Mapping was performed through RSEM software with two mismatches as the bowtie parameter ( $\mathrm{Li}$ and Dewey, 2011). RPKM is the most common method to estimate gene expression level. Furthermore, all the read count numbers of each sample mapping to each gene were normalized to RPKM to calculate gene RPKM values. The 49,340 unigenes had an estimated RPKM value of over 0.3. In particular, 26 unigenes had RPKM values of over 1000, the largest group comprised 28631 unigenes with RPKM values between 1 and 100 , followed by the group with RPKM values between 0.3 and 1 (Table 6). Additionally, RPKM density distribution of J. regia is shown in Figure 6. For the candidate genes involved in pigment biosynthesis, some key enzymes in anthocyanin biosynthesis, such as flavonol 3-O-glucosyltransferase, flavonol 3-O-methyltransferase, flavonoid 3',5'-hydroxylase, flavonol synthase, and chalcone synthase, had high RPKM values (up to 300 to 700). Most genes in the carotenoid pathway had RPKM values between 20 and 100. Only one gene, annotated as 4,5-DOPA dioxygenase for betalain accumulation, had an RPIKM value of 36.89, thereby suggesting that all these three pathways were highly expressed in J. regia red leaves (Additional File 2). Chlorophyll biosynthesis interruption was not the cause of red-leaf trait, but rather the high ratio of anthocyanin, carotenoid, and betalain biosyntheses. 
Table 6. The read counts_RPKM of J. regia genes

\begin{tabular}{ll}
\hline RPKM value & Number \\
\hline$<0.3$ & $4442(8.26 \%)$ \\
$\geq 0.3$ and $<1$ & $19764(36.75 \%)$ \\
$\geq 1$ and $<100$ & $28631(53.23 \%)$ \\
$\geq 100$ and $<1000$ & $919(1.7 \%)$ \\
$\geq 1000$ & $26(0.07 \%)$ \\
Total & 53782 \\
\hline
\end{tabular}

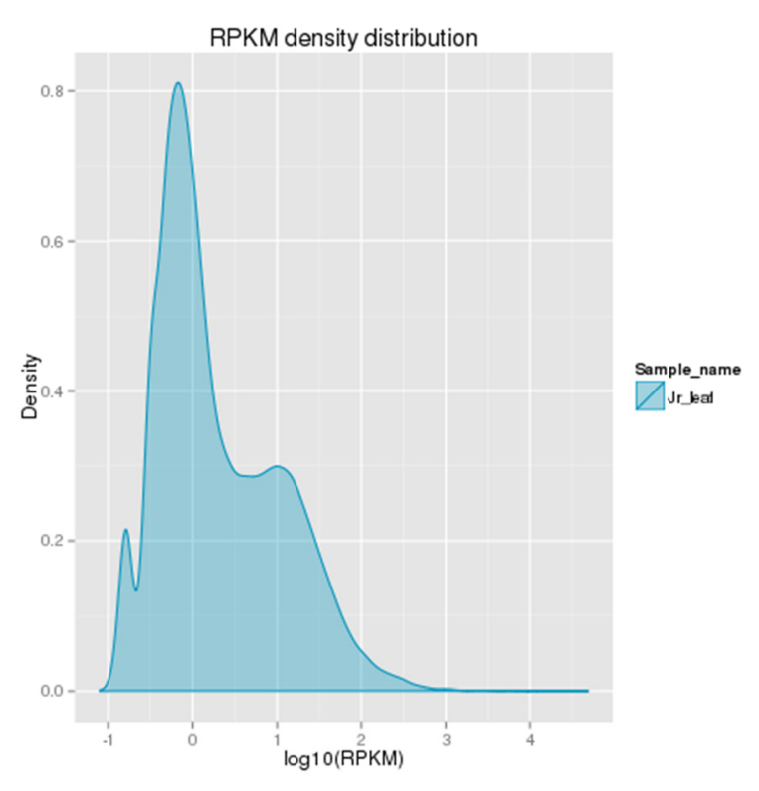

Figure 6. The RPKM density distribution of J. regia transcriptome

\section{Conclusions}

High throughput SSRs, SNPs, and InDel discoveries have been developed with the advent of NGS technology. NGS is an efficient approach for marker discovery and gene mining and for determination of the metabolism pathways involved, especially in species without a reference genome (Strickler et al., 2012). The current study generated 53,782 unigenes, 13,981 SSRs, 41,088 SNPs, and 5,860 InDels. Marker-assisted selection has played an important role in accelerating agriculture breeding. Furthermore, based on the transcriptome data, five pigments related to biosynthetic pathways were identified, and most genes involved in 
anthocyanin and carotenoid biosynthesis were identified, thereby showing that anthocyanins and carotenoids may play essential roles in the development of the red-leaf trait in J. regia. The pigments of plants are controlled by both structural genes and regulatory genes. Some candidate transcription factors were collected. The current study is the first comprehensive report on the large scale transcriptome sequencing analysis of $J$. regia for gene annotation and molecular marker discovery. Considering the reports on the resistance to insects and pathogen of red-leaf, this study provides resources for red-leaf trees breeding.

\section{Acknowledge}

This work was supported by Taian Good Cultivar Engineering Project-Fruit Germplasm Resources Collection and New Germplasm Innovation program (2015-09) to XC, Shandong Good Cultivar Engineering Project-Fruit Germplasm Resources Collection and New Germplasm Innovation program (2014-96) to QS and National Science \& Technology Infrastructure Program(2015-048) of QL.

\section{Abbreviation}

SSR: simple sequence repeats

SNPs: single nucleotide polymorphism

Bp: base pair

bHLH: basic helix-loop-helix

ESTs: expressed sequencing tags

GO: gene ontology

InDels: insertions and deletions

KEGG: Kyoto Encyclopedia of Genes and Genomes

KO: KEGG Ortholog

KOG: Clusters of Orthologous Groups of proteins

$\mathrm{Nr}$ : NCBI non-redundant protein sequences

Nt: NCBI non-redundant nucleotide sequences

Pfam: Protein family

PIFs: phytochrome-interacting factors

RPKM: Reads Per Kilo bases per Million mapped Reads

TFs: Transcription Factors

4,5-DOPA dioxygenase: 4,5-dihydroxyphenylalanine dioxygenase 


\section{References}

Abugri, D. A., Tiimob, B. J., Apalangya, V. A., Pritchett, G., \& McElhenney, W. H. (2013). Bioactive and nutritive compounds in Sorghum bicolor (Guinea corn) red leaves and their health implication. Food Chem, 138, 718-723. https://doi.org/10.1016/j.foodchem.2012.09.149

Albert, N. W., Griffiths, A. G., Cousins, G. R., Verry, I. M., \&d Williams, W. M. (2015). Anthocyanin leaf markings are regulated by a family of R2R3-MYB genes in the genus Trifolium. The New phytologist, 205, 882-893. https://doi.org/10.1111/nph.13100

Amaral, J. S., Valentao, P., Andrade, P. B., Martins, R. C., \& Seabra, R. M. (2008). Do cultivar, geographical location and crop season influence phenolic profile of walnut leaves? Molecules (Basel, Switzerland), 13, 1321-1332. https://doi.org/10.3390/molecules13061321

Cai, C., Zhang, X., Niu, E., Zhao, L., Li, N., Wang, L., Ding, L., \& Guo, W. (2014). GhPSY, a phytoene synthase gene, is related to the red plant phenotype in upland cotton (Gossypium hirsutum L.). Molecular biology reports, 41, 4941-4952. https://doi.org/10.1007/s11033-014-3360-X

Grabherr, M. G., Haas, B. J., Yassour, M., Levin, J. Z., Thompson, D. A., Amit, I., ... Zeng, Q. (2011). Full-length transcriptome assembly from RNA-Seq data without a reference genome. Nature biotechnology, 29, 644-652. https://doi.org/10.1038/nbt.1883

Grotewold, E. (2006). The genetics and biochemistry of floral pigments. Annual review of plant biology, 57, 761-780. https://doi.org/10.1146/annurev.arplant.57.032905.105248

Hosseini, S., Huseini, H. F., Larijani, B., Mohammad, K., Najmizadeh, A., Nourijelyani, K., \& Jamshidi, L. (2014a). The hypoglycemic effect of Juglans regia leaves aqueous extract in diabetic patients: A first human trial. Daru: journal of Faculty of Pharmacy, Tehran University of Medical Sciences, 22, 19. https://doi.org/10.1186/2008-2231-22-19

Hosseini, S., Jamshidi, L., Mehrzadi, S., Mohammad, K., Najmizadeh, A. R., Alimoradi, H., \& Huseini, H. F. (2014b). Effects of Juglans regia L. leaf extract on hyperglycemia and lipid profiles in type two diabetic patients: a randomized double-blind, placebo-controlled clinical trial. Journal of ethnopharmacology, 152, 451-456. https://doi.org/10.1016/j.jep.2014.01.012

Jung, C. S., Griffiths, H. M., De Jong, D. M., Cheng, S., Bodis, M., Kim, T. S., \& De Jong, W.S. (2009). The potato developer (D) locus encodes an R2R3 MYB transcription factor that regulates expression of multiple anthocyanin structural genes in tuber skin. TAG Theoretical and applied genetics Theoretische und angewandte Genetik, 120, 45-57. https://doi.org/10.1007/s00122-009-1158-3

Kaya, H. B., Cetin, O., Kaya, H., Sahin, M., Sefer, F., Kahraman, A., \& Tanyolac, B. (2013). SNP discovery by illumina-based transcriptome sequencing of the olive and the genetic characterization of Turkish olive genotypes revealed by AFLP, SSR and SNP markers. PloS one, 8, e73674. https://doi.org/10.1371/journal.pone.0073674

Li, B., \& Dewey, C. N. (2011). RSEM: accurate transcript quantification from RNA-Seq data 
with or without a reference genome. BMC bioinformatics, 12, 323. https://doi.org/10.1186/1471-2105-12-323

McKenna, A., Hanna, M., Banks, E., Sivachenko, A., Cibulskis, K., Kernytsky, A., ... Daly, M. (2010). The Genome Analysis Toolkit: a MapReduce framework for analyzing next-generation DNA sequencing data. Genome research, 20, 1297-1303. https://doi.org/10.1101/gr.107524.110

Mouradov, A., \& Spangenberg, G. (2014). Flavonoids: a metabolic network mediating plants adaptation to their real estate. Frontiers in plant science, 5, 620. https://doi.org/10.3389/fpls.2014.00620

Nael Abu Taha, M. A. A. -w. (2011). Utility and importance of walnut, Juglans regia Linn: A review. Afr J Microbiol Res, 5, 10. https://doi.org/10.5897/AJMR11.610

Paudel, P., Satyal, P., Dosoky, N. S., Maharjan, S., \& Setzer, W. N. (2013). Juglans regia and J. nigra, two trees important in traditional medicine: A comparison of leaf essential oil compositions and biological activities. Natural product communications, 8', 1481-1486.

Pereira, J. A., Oliveira, I., Sousa, A., Valentao, P., Andrade, P. B., Ferreira, I. C., ... Estevinho, L. (2007). Walnut (Juglans regia L.) leaves: phenolic compounds, antibacterial activity and antioxidant potential of different cultivars. Food and chemical toxicology : an international journal published for the British Industrial Biological Research Association, 45, 2287-2295.

Qi, J. X., H.Y., Zhu, Y, \& Wu, C. L. (2011). Studies on germplasm of Juglans by EST-SSR markers. Acta Hortic Sin, 38, 8.

Qureshi, M. N., Stecher, G., \& Bonn, G. K. (2014). Determination of total polyphenolic compounds and flavonoids in Juglans regia leaves. Pakistan journal of pharmaceutical sciences, 27, 865-869.

Ranjan, S., Singh, R., Singh, M., Pathre, U. V., \& Shirke, P. A. (2014). Characterizing photoinhibition and photosynthesis in juvenile-red versus mature-green leaves of Jatropha curcas L. Plant physiology and biochemistry : $P P B$ / Societe francaise de physiologie vegetale, 79, 48-59. https://doi.org/10.1016/j.plaphy.2014.03.007

Rodriguez-Villalon, A., Gas, E., \& Rodriguez-Concepcion, M. (2009). Phytoene synthase activity controls the biosynthesis of carotenoids and the supply of their metabolic precursors in dark-grown Arabidopsis seedlings. The Plant journal : for cell and molecular biology, 60, 424-435. https://doi.org/10.1111/j.1365-313X.2009.03966.x

Sandmann, G., Romer, S., \& Fraser, P. D. (2006). Understanding carotenoid metabolism as a necessity for genetic engineering of crop plants. Metabolic engineering, 8, 291-302. https://doi.org/10.1016/j.ymben.2006.01.005

Strickler, S. R., Bombarely, A., \& Mueller, L. A. (2012). Designing a transcriptome next-generation sequencing project for a nonmodel plant species. Americam journal of botany, 99, 257-266. https://doi.org/10.3732/ajb.1100292 


\section{MInstitute Macrothink $_{\text {Int }}$}

Tanaka, Y., Sasaki, N., \& Ohmiya, A. (2008). Biosynthesis of plant pigments: anthocyanins, betalains and carotenoids. The Plant journal : for cell and molecular biology, 54, 733-749. https://doi.org/10.1111/j.1365-313X.2008.03447.x

Tian, J., Peng, Z., Zhang, J., Song, T., Wan, H., Zhang, M., \& Yao, Y. (2015). McMYB10 regulates coloration via activating $\mathrm{McF}^{\prime} \mathrm{H}$ and later structural genes in ever-red leaf crabapple. Plant biotechnology journal. https://doi.org/10.1111/pbi.12331

Wang, Y. S., Liu, Z. Y., Li, Y. F., Zhang, Y., Yang, X. F., \& Feng, H. (2013). Identification of sequence-related amplified polymorphism markers linked to the red leaf trait in ornamental kale (Brassica oleracea L. var. acephala). Genetics and molecular research : GMR, 12, 870-877. https://doi.org/10.4238/2013.April.2.3

Wu, J., Gu, Y. Q., Hu, Y., You, F. M., Dandekar, A. M., Leslie, C. A.,... Luo, M. C. (2012). Characterizing the walnut genome through analyses of BAC end sequences. Plant molecular biology, 78, 95-107. https://doi.org/10.1007/s11103-011-9849-y

Yang, Y. N., Yao, G. F., Zheng, D., Zhang, S. L., Wang, C., Zhang, M. Y., \& Wu, J. (2015). Expression differences of anthocyanin biosynthesis genes reveal regulation patterns for red pear coloration. Plant cell reports, 34, 189-198. https://doi.org/10.1007/s00299-014-1698-0

Zhang R, Z.A., \& Wang, X. J, Yu, J. (2010). Development of Juglans regia SSR markers by data mining of the EST database. Plant Mol Biol Rep, 28, 9. https://doi.org/10.1007/s11105-010-0192-2

Zhang, Z. Y., Han, J. W., Jin, Q., Wang, Y., Pang, X. M., \& Li, Y. Y. (2013)). Development and characterization of new microsatellites for walnut (Juglans regia). Genetics and molecular research : GMR, 12, 4723-4734. https://doi.org/10.4238/2013.October.18.10

Zhao, M. H., Jiang, Z. T. L. (2014). Flavonoids in Juglans regia L. leaves and evaluation of in vitro antioxidant activity via intracellular and chemical methods. The Scientific World Journal, 303878.

Zhou, Y., Zhou, H., Lin-Wang, K., Vimolmangkang, S., Espley, R. V., Wang, L., Allan, A C., \& Han, Y. (2014). Transcriptome analysis and transient transformation suggest an ancient duplicated MYB transcription factor as a candidate gene for leaf red coloration in peach. BMC plant biology, 14, 388. https://doi.org/10.1186/s12870-014-0388-y

Zhu, M., Chen, G., Zhou, S., Tu, Y., Wang, Y., Dong, T., \& Hu, Z. (2014). A new tomato NAC (NAM/ATAF1/2/CUC2) transcription factor, SINAC4, functions as a positive regulator of fruit ripening and carotenoid accumulation. Plant \& cell physiology, 55, 119-135. https://doi.org/10.1093/pcp/pct162 
Additional File 1. The lengths distributions of transcripts and unigenes

\begin{tabular}{llllll}
\hline Transcript length interval & $\mathbf{2 0 0 - 5 0 0 b p}$ & $\mathbf{5 0 0 - 1 k b p}$ & $\mathbf{1 k - 2 k b p}$ & $\mathbf{2} \mathbf{2 k b p}$ & - \\
\hline $\begin{array}{l}\text { Total } \\
\text { Number of transcripts }\end{array}$ & 38000 & 17954 & 24702 & 19949 & 100605 \\
\hline $\begin{array}{l}\text { Total } \\
\text { Number of Unigenes }\end{array}$ & 31833 & 8950 & 7600 & 5399 & 53782 \\
\hline
\end{tabular}

Additional File 2. Candidate genes related to the pigmentation of Juglans regia red-leaf

\begin{tabular}{|c|c|c|c|c|c|}
\hline Function & Unigene No. & KO & Enzyme & $\begin{array}{l}\text { Pathway } \\
\text { ID }\end{array}$ & RPKM \\
\hline \multirow{12}{*}{$\begin{array}{l}\text { Porphyrin } \\
\text { and } \\
\text { chlorophyll } \\
\text { metabolism }\end{array}$} & comp14200_c0 & K02257 & protoheme IX farnesyltransferase & \multirow[t]{12}{*}{ ko00860 } & 22.50 \\
\hline & comp20631_c0 & K01749 & hydroxymethylbilane synthase & & 50.42 \\
\hline & comp10637_c0 & K02259 & $\begin{array}{l}\text { cytochrome c oxidase assembly protein } \\
\text { subunit } 15\end{array}$ & & 11.98 \\
\hline & comp29312_c0 & K01845 & $\begin{array}{l}\text { glutamate-1-semialdehyde } \\
\text { 2,1-aminomutase }\end{array}$ & & 88.33 \\
\hline & comp25659_c0 & K02492 & glutamyl-tRNA reductase & & 134.71 \\
\hline & comp9774_c0 & K02492 & glutamyl-tRNA reductase & & 15.85 \\
\hline & comp10356_c0 & K02492 & glutamyl-tRNA reductase & & 51.31 \\
\hline & comp32259_c0 & K02492 & glutamyl-tRNA reductase & & 21.01 \\
\hline & comp10356_c1 & K02492 & glutamyl-tRNA reductase & & 59.13 \\
\hline & comp18909_c0 & K00522 & ferritin heavy chain & & 17.34 \\
\hline & comp18909_c1 & K00522 & ferritin heavy chain & & 8.11 \\
\hline & comp20893_c0 & K00522 & ferritin heavy chain & & 58.53 \\
\hline
\end{tabular}


comp21097_c0 K04040 chlorophyll synthase

comp125945_c0 K04040 chlorophyll synthase

comp23862_c0 K00228 coproporphyrinogen III oxidase

comp1031_c0 K03403 magnesium chelatase subunit H

comp5337_c0 K03403 magnesium chelatase subunit H

comp170589_c0 K03403 magnesium chelatase subunit H

comp28686_c0 K03403 magnesium chelatase subunit H

comp17992_c0 K01698 porphobilinogen synthase

comp11188_c0 K10960 geranylgeranyl reductase

comp28845_c0 K10960 geranylgeranyl reductase

201.57

comp25852_c1 K08101 $\begin{aligned} & \text { phytochromobilin:ferredoxin } \\ & \text { oxidoreductase }\end{aligned}$

comp26939_c0 K13600 chlorophyllide a oxygenase

comp11746_c0 K13600 chlorophyllide a oxygenase

comp25409_c0 K13606 chlorophyll(ide) b reductase

comp27579_c0 K01772 ferrochelatase

comp16739_c0 K01772 ferrochelatase

comp26150_c0 K01719 uroporphyrinogen-III synthase

comp30546_c0 K01599 uroporphyrinogen decarboxylase

comp24500_c0 K01599 uroporphyrinogen decarboxylase

comp215367_c0 K01599 uroporphyrinogen decarboxylase 


\begin{tabular}{|c|c|c|c|c|c|}
\hline \multirow{4}{*}{$\begin{array}{l}\text { Carotenoid } \\
\text { biosynthesis }\end{array}$} & comp14630_c2 & K00218 & protochlorophyllide reductase & \multirow{4}{*}{ ko00906 } & 11.28 \\
\hline & comp14630_c1 & K00218 & protochlorophyllide reductase & & 15.98 \\
\hline & comp26309_c1 & K15746 & beta-carotene 3-hydroxylase & & 82.90 \\
\hline & comp31739_c0 & K15746 & beta-carotene 3-hydroxylase & & 12.70 \\
\hline & comp10365_c0 & K15747 & $\begin{array}{l}\text { cytochrome P450, family } 97 \text {, subfamily } \\
\text { A (beta-ring hydroxylase) }\end{array}$ & & 44.35 \\
\hline & comp25896_c0 & K15744 & zeta-carotene isomerase & & 9.58 \\
\hline & comp9456_c0 & K09843 & $(+)$-abscisic acid 8'-hydroxylase & & 4.74 \\
\hline & comp1748_c0 & K09843 & $(+)$-abscisic acid 8'-hydroxylase & & 1.21 \\
\hline & comp25736_c0 & K09843 & $(+)$-abscisic acid 8'-hydroxylase & & 17.98 \\
\hline & comp13144_c0 & K09843 & $(+)$-abscisic acid 8'-hydroxylase & & 1.15 \\
\hline & comp25328_c1 & K09843 & $(+)$-abscisic acid 8'-hydroxylase & & 47.66 \\
\hline & comp27108_c2 & K09843 & $(+)$-abscisic acid 8'-hydroxylase & & 1.25 \\
\hline & comp80215_c0 & K09843 & $(+)$-abscisic acid 8'-hydroxylase & & 25.02 \\
\hline & comp13725_c1 & K09843 & $(+)$-abscisic acid 8'-hydroxylase & & 25.02 \\
\hline & comp25736_c0 & K09843 & $(+)$-abscisic acid 8'-hydroxylase & & 17.98 \\
\hline & comp13144_c0 & K09843 & $(+)$-abscisic acid 8'-hydroxylase & & 1.15 \\
\hline & comp25328_c1 & K09843 & $(+)$-abscisic acid 8'-hydroxylase & & 6.86 \\
\hline & comp27108_c2 & K09843 & $(+)$-abscisic acid 8'-hydroxylase & & 1.25 \\
\hline & comp80215_c0 & K09843 & $(+)$-abscisic acid 8'-hydroxylase & & - \\
\hline & comp13725_c1 & K09843 & $(+)$-abscisic acid 8'-hydroxylase & & 25.02 \\
\hline & comp10689_c0 & K02291 & phytoene synthase & & 38.17 \\
\hline
\end{tabular}




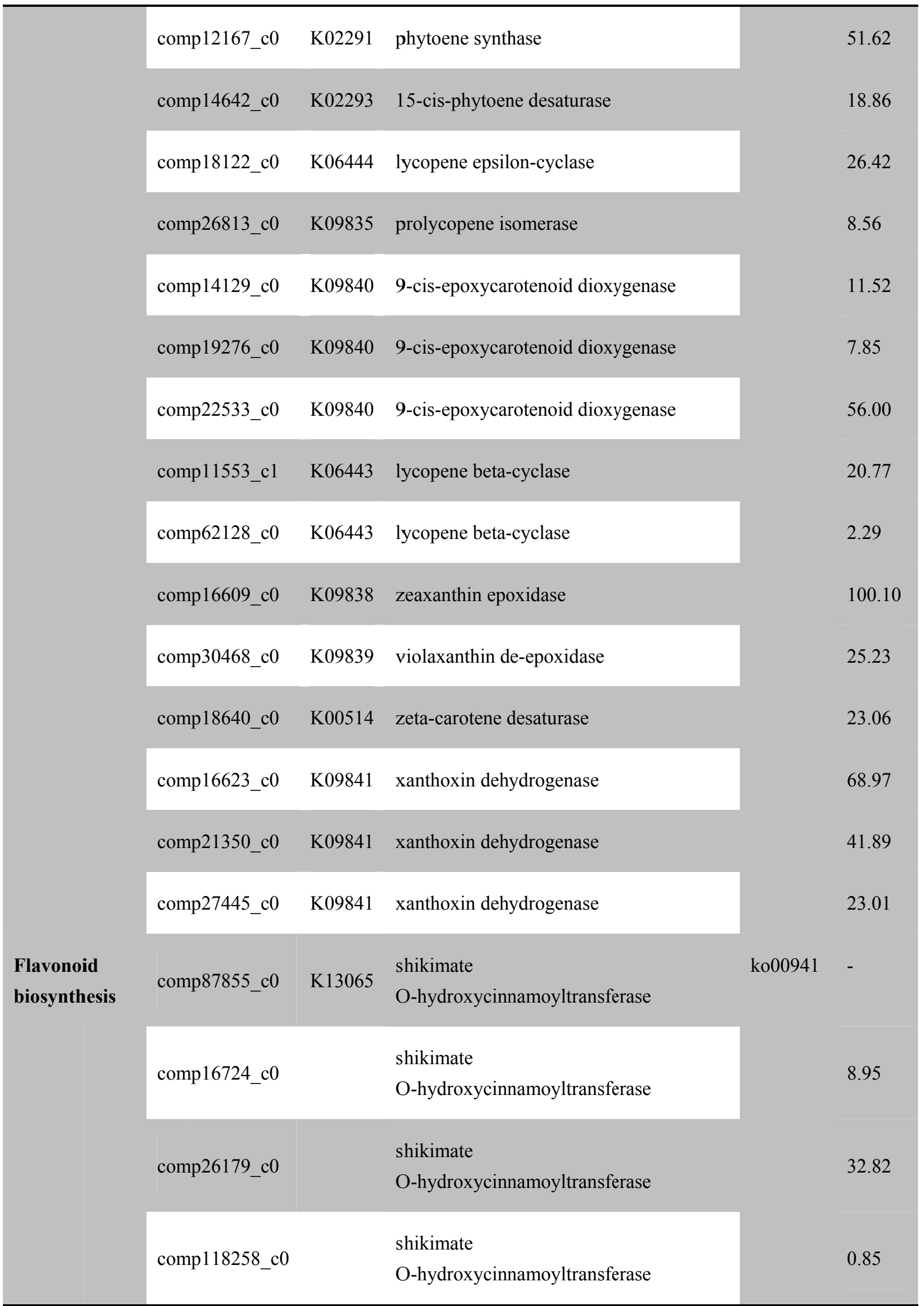




\begin{tabular}{l|l} 
comp182423_c0 & shikimate \\
O-hydroxycinnamoyltransferase
\end{tabular}

comp75175_c0

shikimate

O-hydroxycinnamoyltransferase

comp16079_c0 K08695 anthocyanidin reductase

comp10118_c0 K13083

cytochrome P450, family 75, subfamily

A (flavonoid 3',5'-hydroxylase)

comp29670_c0

K13082

bifunctional

dihydroflavonol

4-reductase/flavanone 4-reductase

comp21359_c0 K13081 leucoanthocyanidin reductase

comp23640_c0

leucoanthocyanidin reductase

comp46728_c0

leucoanthocyanidin reductase

comp22978_c0

leucoanthocyanidin reductase

comp29197_c0 K01859 chalcone isomerase

comp28746_c0 K00475 naringenin 3-dioxygenase

comp20952_c0 K05277 leucoanthocyanidin dioxygenase

306.62

comp25022_c1 K00660 chalcone synthase

767.76

comp10942_c0

chalcone synthase

226.16

comp93311_c0 chalcone synthase

comp41264_c0

chalcone synthase

7.06

comp13594_c0 $\quad$ K09754 $\begin{aligned} & \text { coumaroylquinate(coumaroylshikimate) } \\ & \text { 3'-monooxygenase }\end{aligned}$

comp27087_c0

coumaroylquinate(coumaroylshikimate)

3'-monooxygenase 


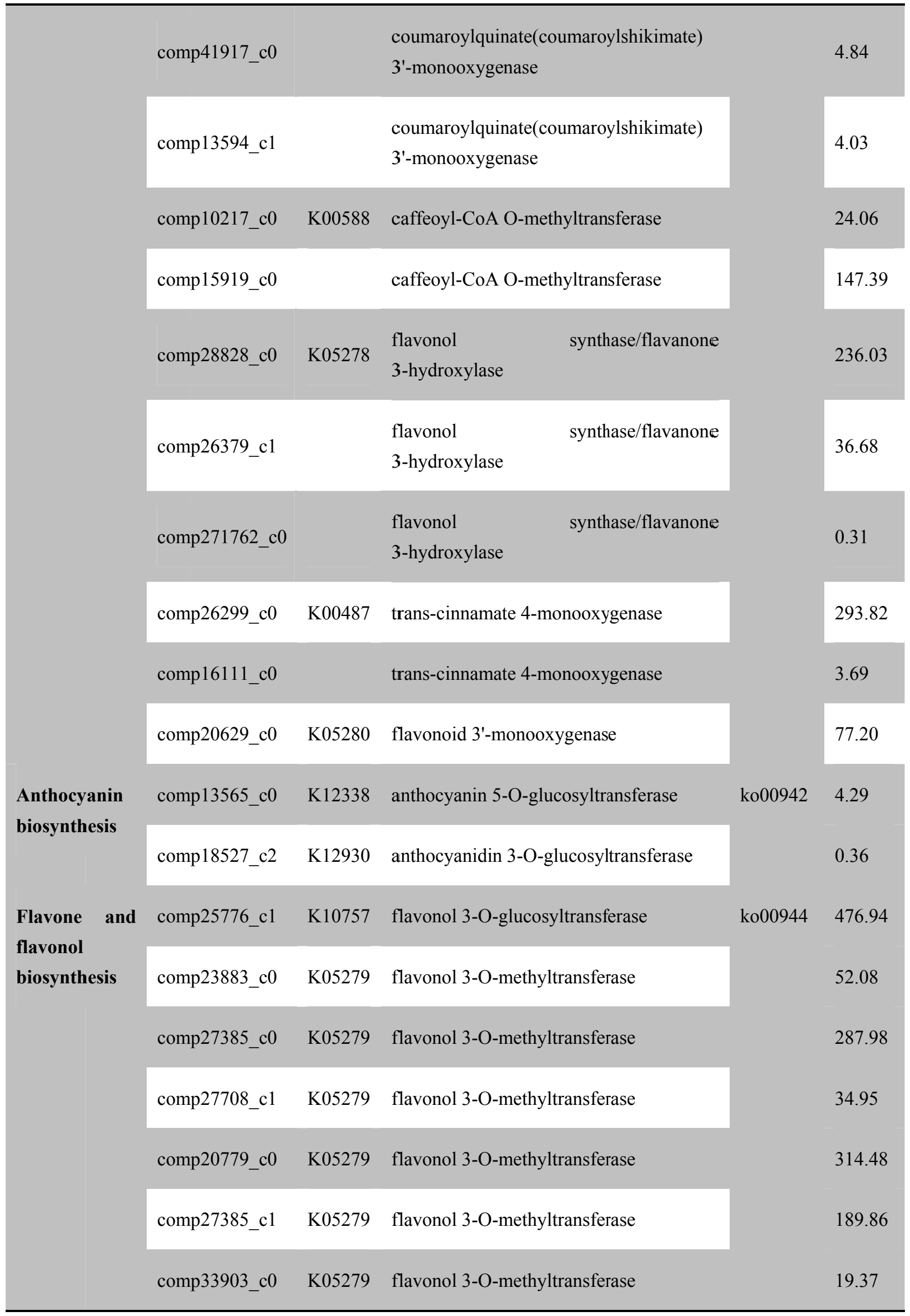




\begin{tabular}{|c|c|c|c|c|c|}
\hline & $4-2=$ & & Journal of Ap & $\begin{array}{c}\text { plied Biot } \\
\text { ISSN } \\
2017 \text {, Vol. }\end{array}$ & $\begin{array}{l}\text { chnology } \\
327-0640 \\
5, \text { No. } 2 \\
\end{array}$ \\
\hline \multirow{5}{*}{$\begin{array}{l}\text { Betalain } \\
\text { biosynthesis }\end{array}$} & comp75470_c0 & K05279 & flavonol 3-O-methyltransferase & \multirow{5}{*}{ ko00965 } & - \\
\hline & comp10118_c0 & K13083 & $\begin{array}{l}\text { cytochrome } \mathrm{P} 450 \text {, family } 75 \text {, subfamily } \\
\text { A (flavonoid 3',5'-hydroxylase) }\end{array}$ & & 235.43 \\
\hline & comp20629_c0 & K05280 & flavonoid 3'-monooxygenase & & 77.20 \\
\hline & comp17877_c0 & K15777 & 4,5-DOPA dioxygenase extradiol & & 36.89 \\
\hline & comp49680_c0 & K15777 & 4,5-DOPA dioxygenase extradiol & & 3.76 \\
\hline
\end{tabular}

\section{Copyright Disclaimer}

Copyright reserved by the author(s).

This article is an open-access article distributed under the terms and conditions of the Creative Commons Attribution license (http://creativecommons.org/licenses/by/3.0/). 